\title{
Multi-Frequency Blazar Micro-Variability as a Tool to Investigate Relativistic Jets
}

\author{
James R. Webb \\ Department of Physics, Florida International University, Miami, FL 33199, USA; webbj@fiu.edu; \\ Tel.: +1-305-348-3964 \\ Academic Editors: Jose L. Gómez, Alan P. Marscher and Svetlana G. Jorstad \\ Received: 29 June 2016; Accepted: 17 August 2016; Published: 29 August 2016
}

\begin{abstract}
For the past 12 years we have been studying optical micro-variability of a sample of 15 Blazars. We summarize the results of this study and draw some basic conclusions about the characteristics of micro-variability. The intermittency, the stochastic nature, and the similar profile shapes seen in micro-variations at different times and in different objects have led us to a possible model to explain the observed micro-variations. The model is based on a strong shock propagating down a relativistic jet and encountering turbulence which causes density or magnetic field enhancements. We use the theory of Kirk, Reiger, and Mastichiadis (1998) to describe the pulse of synchrotron emission emanating from individual density enhancements energized by the shock. By fitting these "pulses" to micro-variability observations, we obtain excellent fits to actual micro-variations. The model predicts that the spectral index changes as a function of pulse duration. This effect should be observable in multi-frequency micro-variability data. We present the theoretical model, model fits of our micro-variability light curves, and preliminary multi-frequency micro-variability observations that support this model. A further test that has yet to be carried out involves observing polarization changes in different pulses.
\end{abstract}

Keywords: Blazar; optical micro-variability; turbulent jets

\section{Introduction}

Micro-variations are very short-term, low amplitude variations in the optical part of the spectrum. These variations have been studied by a number of observers but no convincing model to account for the characteristics of these variations has been found. Our program at Florida International University has observed micro-variability in a sample of fifteen Blazars during 164 nights over 12 years. We define micro-variability as: "Rapid, low-amplitude non-linear variations in optical fluctuations in a single night". The observed timescales are from a few minutes to a few hours, and amplitudes range from 0.01 to 0.1 magnitudes. Figure 1 is an example of micro-variations observed over a single night.

The question becomes: What are the possible explanations of this type of variability? The cause could be extrinsic to the source, perhaps by interstellar scintillation or detector effects such as drifting gains in the CCD or local weather disturbances. Interstellar scintillation can be ruled out because the timescales are far too rapid for optical interstellar scintillation and two separate telescopes should observe different waveforms [1]. Observations by Pollock, Webb and Azarnia [2] showed that two different telescopes at two sites with different equipment, different weather, and a different vantage point show the same variations. This rules out both interstellar scintillation and detector/local effects leaving intrinsic effects as the origin of these rapid variations. Since these objects are jet dominated and very little disk emission is visible during outbursts, we consider jet processes as the underlying cause. This paper discusses the results of our program and outlines our attempts to provide an explanation for the observed characteristics of these micro-variations. 


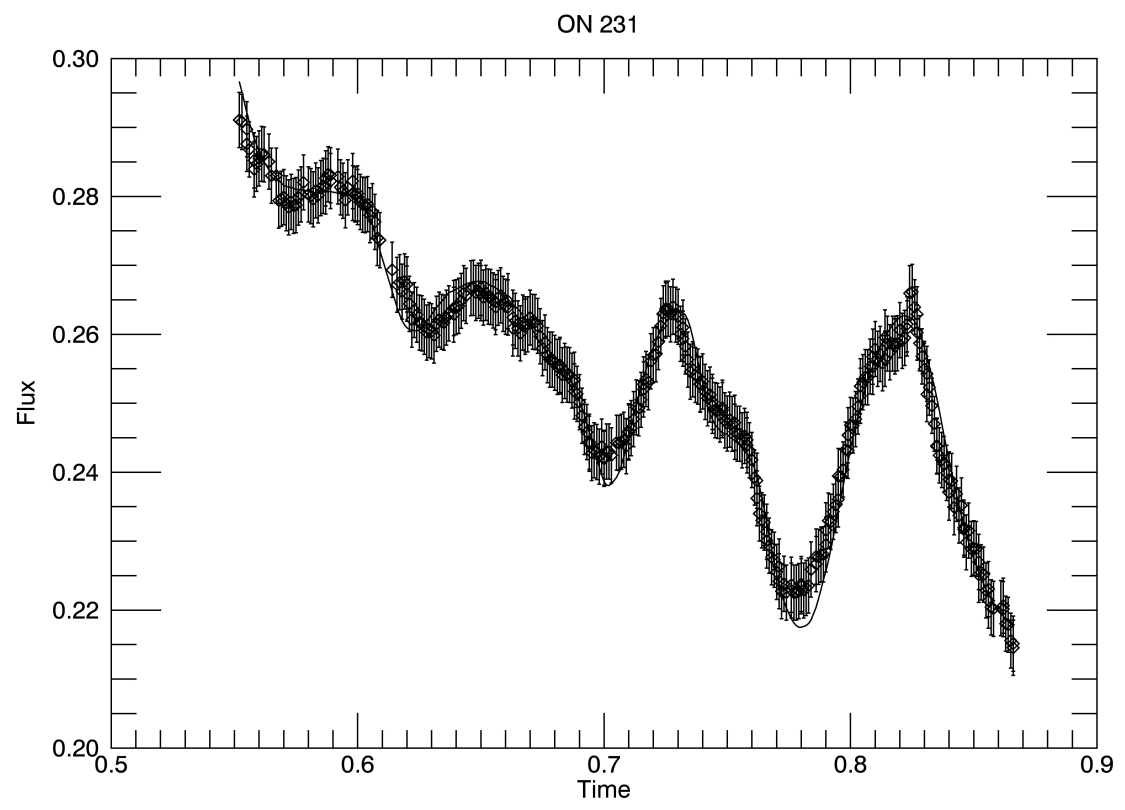

Figure 1. The micro-variability light curve of ON 231 taken over a single night.

\section{Materials and Methods}

The Florida International Blazar group led by the author involves observations made with the 0.9 m SARA telescope at Kitt Peak National Observatory (KPNO), the $24^{\prime \prime}$ Lowell telescope at Cerro Tololo Interamerican Observatory (CTIO) and the $1 \mathrm{~m}$ Jacobus Kaptyne telescope at the Roque de Los Muchachos Observatory (RM). Additional observations were made with $24^{\prime \prime}$ telescope at the Florida International University's Stocker Astroscience center. The research involves students at all levels, including high school interns. This paper reports on work from a variety of students, past and present: Gopal Bhatta, now at Jagiellonian University, Douglas Laurence (current graduate student), Sarah Dhalla former graduate student, and undergraduates, Steven Revesz, Daniel Puentes, Carmen Vargas, Jordan DeWitt, and Archit Khanuja'Aad. High school intern Gabriel Salazar also contributed to data reduction. The observations made at the remote sites were done from the FIU advanced starship telescope control room. Figure 2 shows the control room set up for remote observing. The monitors lining the front wall are all switchable; i.e., any computer output can easily be placed on the main screen or any one of the subsidiary screens. Each remote telescope has its own station and series of monitors which monitor telescope operations, camera outputs and weather. All of the computers are actually located in a computer room off the main control room, and only the monitors, keyboards and mice are in the room. The room is equipped with sidereal clocks for each telescope location, comfortable chairs on wheels which facilitates long duration observing sessions. A complete surround sound/Blue ray entertainment system enhances the observing experience and is used for teaching and demonstrations. The furniture in the room was custom built to resemble a starship bridge and the round room is decorated with Star Fleet command logo. It also has recessed lighting in the vertical panels and the circular ceiling to more closely resemble a starship bridge. The room has proved to be optimized and efficient for operation of up to four remote telescopes simultaneously by one or two people. It is also large enough to accommodate up to 20 people for classroom use. The $24^{\prime \prime}$ computerized telescope on the roof is used for teaching and research, while the three SARA remotely accessible telescopes are used primarily for research.

The observations consisted of regular monitoring of several sources at a cadence of one or two observations per month. Objects were chosen to do micro-variability observations based on weather, brightness, and activity considerations. We attempted to obtain a micro-variability observation of each object over its entire magnitude range: faint, intermediate and flare brightness 
levels. The objects involved in this study are described in Table 1. Column 1 lists the objects common name while column 2 shows which classification is normally associated with the source. Column 3 lists the redshift while columns 4 and 5 give the number of nights observed and the number of micro-variability runs done for that object. The final column gives the computed duty cycle for the source. The duty cycle is simply the time the object showed micro-variability divided by the time total time it was observed. Note the largest duty cycle is for S5 $0716+71$ at around $86 \%$.

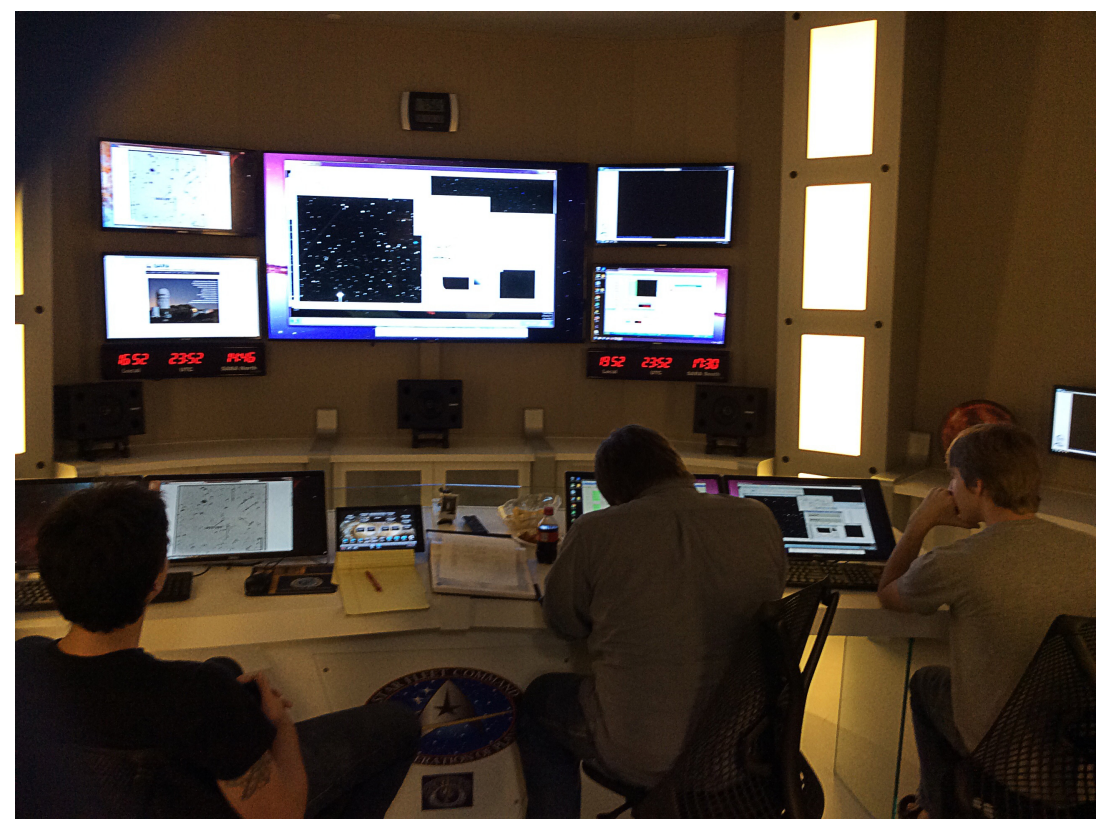

Figure 2. Starship control room being used to control remote telescopes at Kitt Peak National Observatory (KPNO) and Cerro Tololo Interamerican Observatory (CTIO).

Table 1. Source list for the FIU Blazar Micro-variability Program.

\begin{tabular}{cccccc}
\hline Object & Type & Z & Nights Observed & Micro-Variability Runs & Duty Cycle \\
\hline AO 0235+164 & BL Lac & 0.940 & 59 & 14 & 54.66 \\
PKS0420-01 & OVV & 0.916 & 31 & 10 & 15.97 \\
S5 0716+71 & BL Lac & 0.300 & 47 & 36 & 85.93 \\
0735+017 & BL Lac & 0.424 & 15 & 2 & 00.00 \\
0736+017 & OVV & 0.189 & 4 & 3 & 11.29 \\
OJ 287 & BL Lac & 0.306 & 28 & 17 & 49.00 \\
1156+295 & OVV & 0.725 & 16 & 6 & 50.99 \\
3C 273 & QSO & 0.158 & 5 & 2 & 25.28 \\
3C 279 & OVV & 0.536 & 28 & 9 & 61.07 \\
1510-01 & OVV & 0.350 & 13 & 8 & 64.70 \\
3C 345 & OVV & 0.593 & 31 & 12 & 33.83 \\
ON 231 & BL Lac & 0.102 & 4 & 3 & 29.87 \\
3C 454.3 & OVV & 0.859 & 9 & 7 & 56.54 \\
BL Lac & BL Lac & 0.068 & 77 & 31 & 54.73 \\
3C 446 & OVV & 1.404 & 20 & 4 & 42.86 \\
\hline
\end{tabular}

The uneven sampling among the different sources reflects several observational biases. For historically faint sources, micro-variability observations must be done when the object is accessible, on superior photometric nights. For brighter sources, the weather requirements were less stringent and thus these sources were much more heavily observed. In addition, sources with higher duty cycles tended to be more heavily observed since we wanted to model the micro-variability in these sources. Figure 3 shows examples of the light curves of our sources with black data points 
indicating a single night's brightness measurement, green up-pointing arrows indicating a positive detection of micro-variations for that night, and a down pointed red arrow indicating a negative micro-variability detection.
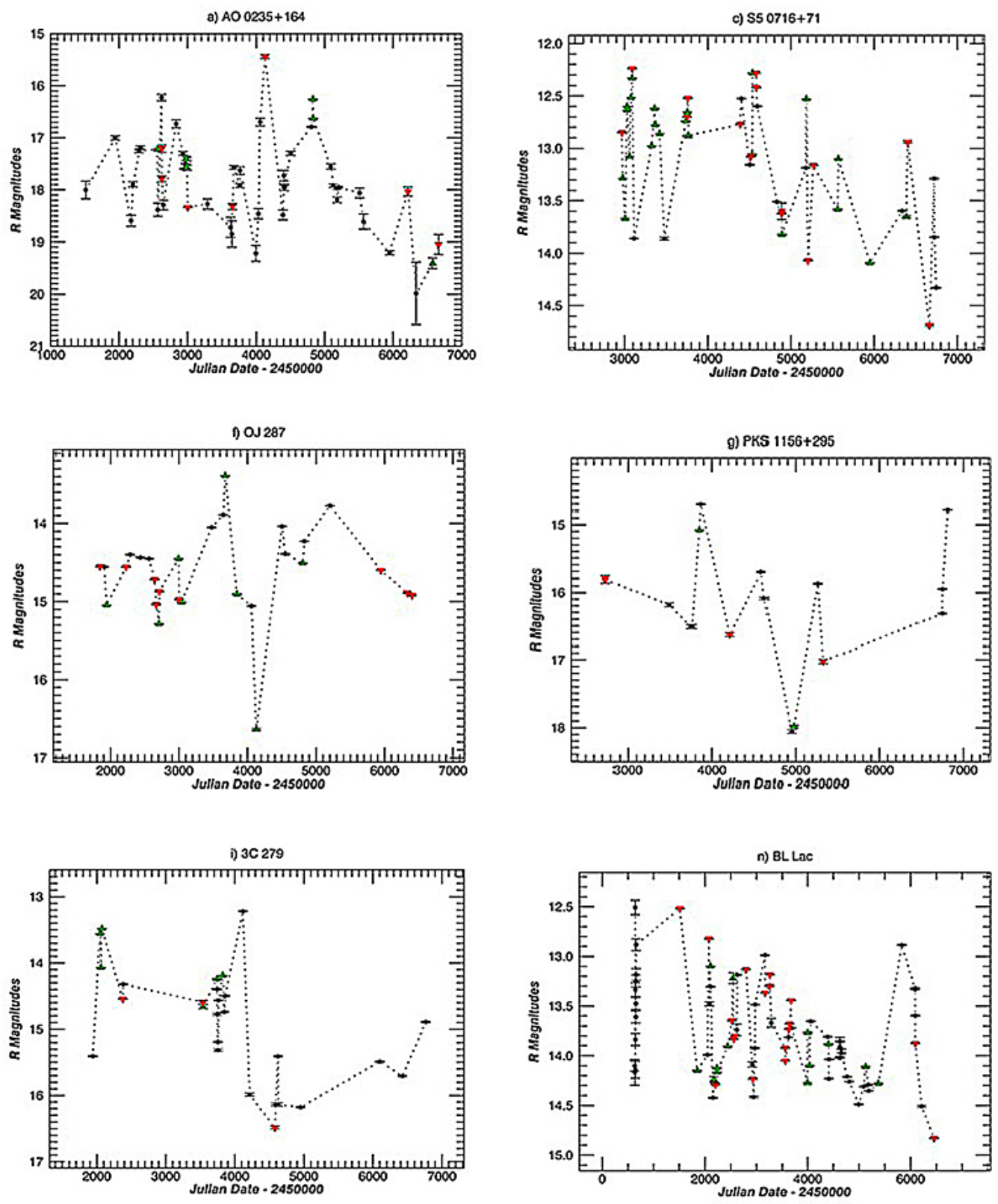

Figure 3. Long-term light curves of six of the sources from Table 1. The objects are in clockwise order: AO 0235+164, S5 0716+71, PKS 1156+295, BL Lac, 3C 279, and OJ 287. Green up-pointing arrows indicate that micro-variability was seen during that observation. The red down-pointing arrows indicated that no micro-variability was seen during the night. Note that for these six objects in particular, the green and red arrows both encompass the entire range of brightness and do not appear to be correlated with brightness level.

\section{Results}

The results of our intensive study revealed the following observational characteristics of micro-variability seen in this sample. Micro-variations are intermittent, showing a large range of duty 
cycles among the different objects. The duty cycles range from 85.93 percent for BL Lac S5 0716+71 to $11 \%$ for OVV quasar PKS $0736+017$ (objects with more than 3 micro-variability observations). The presence of micro-variability for the objects in our sample are not statistically correlated with overall brightness [3,4]. Analysis of our observations with time series analysis techniques such as DFT, Wavelet and periodograms do not show repeatable timescales for any of these sources [4]. Time series and noise analyses show that the observed micro-variations do not show characteristics of pure noise in the sense that it is unresolved red noise or white noise [5,6]. The ON 231 micro-variability curve in particular seemed to exhibit resolved individual events or "pulses", and this same shape seemed to be present in other sources as well $[3,7]$. We initially fit the bursts or pulses with a Gaussian curves and obtained reasonable fits, but were interested in more physical form for the pulses.

Turbulence in relativistic jets has been used to explain the lack of polarization from synchrotron emission in the jets [8,9]. Turbulence has also been proposed to account for the red noise X-ray PDS in quasars [10]. Recent models to explain the multi-wave variability in Blazars has also invoked the idea of turbulent cells in the relativistic jets [11]. We therefore proposed a model for micro-variations in light of the intermittent nature of micro-variability and the common pulse shapes. This model consists of turbulence in the jet flow, the turbulent cells are then excited by shock waves and cool through synchrotron radiation. In this model, micro-variability is seen if the jet flow is turbulent and the turbulent cells either pass through a standing shock or encounter a moving shock. If the jet flow is laminar, then the shock produces short-term variability but no micro-variability. The observed micro-variability is then the superposition of individual synchrotron radiating turbulent cells. Figure 4 shows a cartoon of the light curve and the possible cell distribution in the jet.

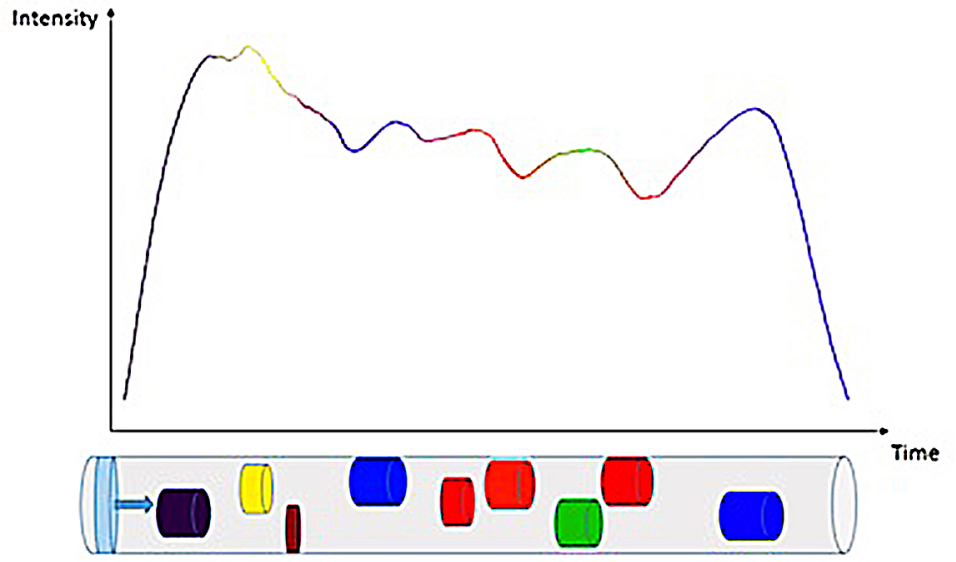

Figure 4. Illustration of a simulated micro-variability curve and a possible distribution of turbulent cells from which the light curve originates. The shock is on the left and propagates down the jet to the right accelerating particles as it encounters the turbulent cells. The radial positions of the cells are arbitrary since we have no depth information.

We use the theory of Kirk et al. [12] to calculate the radiation emitted after the shock energizes the electrons in the turbulent cell. Their work attempted to explain short-term flares as a planar shock encountering a dense zone in a cylindrical jet. They solve the diffusion equations:

$$
\begin{gathered}
\frac{\partial n}{\partial t}-\frac{\partial}{\partial \gamma}\left(\beta_{s} \gamma^{2} n\right)=\frac{N(\gamma, t)}{t_{e s c}} \delta\left(x-x_{s}(t)\right), \\
I_{0}(v, \bar{t})=\int d \gamma P(\nu, \gamma) \int d x n\left(x, \gamma, \bar{t}+\frac{x}{c}\right)
\end{gathered}
$$


These equations were programmed in IDL and solved for the frequencies at which we observe providing pulse shapes for comparison to actual micro-variability light curves. The resulting pulse shape for a set of parameters is shown in Figure 5. The parameters used were: $\mathrm{B}=2$ Gauss, $\mathrm{Q}=609.3 \mathrm{~m}^{3} \mathrm{~s}^{-1}, v=4.3 \times 10^{14}, t_{f}=2.5 / t_{a c c}$, and a shock speed of $0.1 \mathrm{c}$. B is the magnetic field in Gauss, $Q$ is the injection rate, $t_{\mathrm{f}}$ is the flare duration, and $t_{a c c}$ is the acceleration time for electrons.

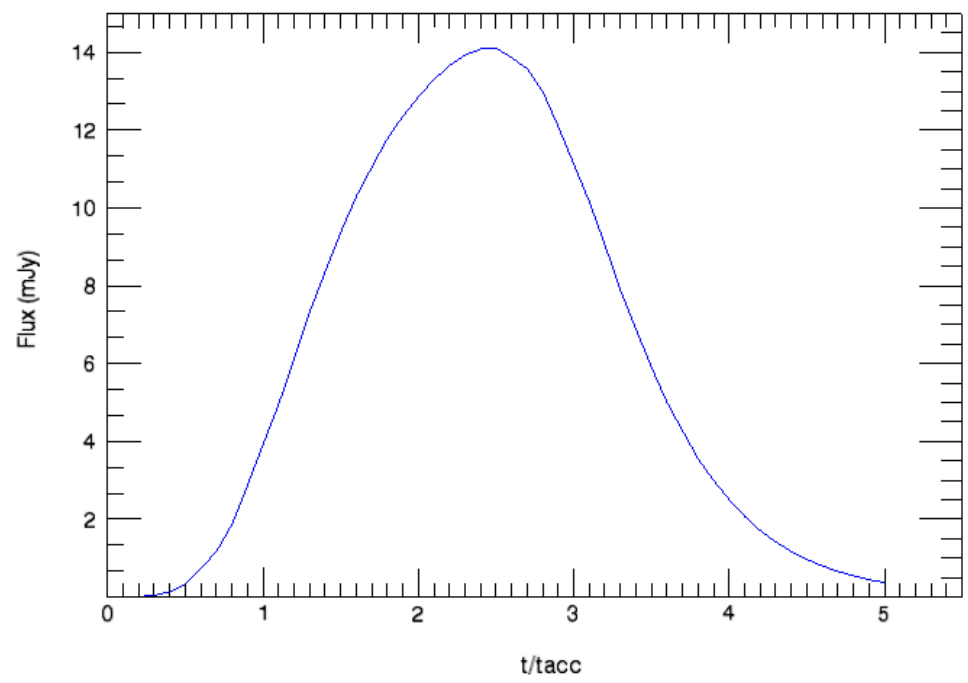

Figure 5. The pulse shape for a shock moving at 1c encountering a density enhancement with the following parameters: $B=2$ Gauss, $Q=609.3 \mathrm{~m}^{3} \mathrm{~s}^{-1}, v=4.3 \times 10^{14}, \mathrm{t}_{\mathrm{f}}=2.5 / \mathrm{t}_{\mathrm{acc}}$.

The maximum gamma of the electrons was assumed to be 10,000. We then scale the pulse in amplitude and duration to fit particular pulses identified in the light curve. Figure 6 shows the process of fitting successive pulses to a micro-variability curve.

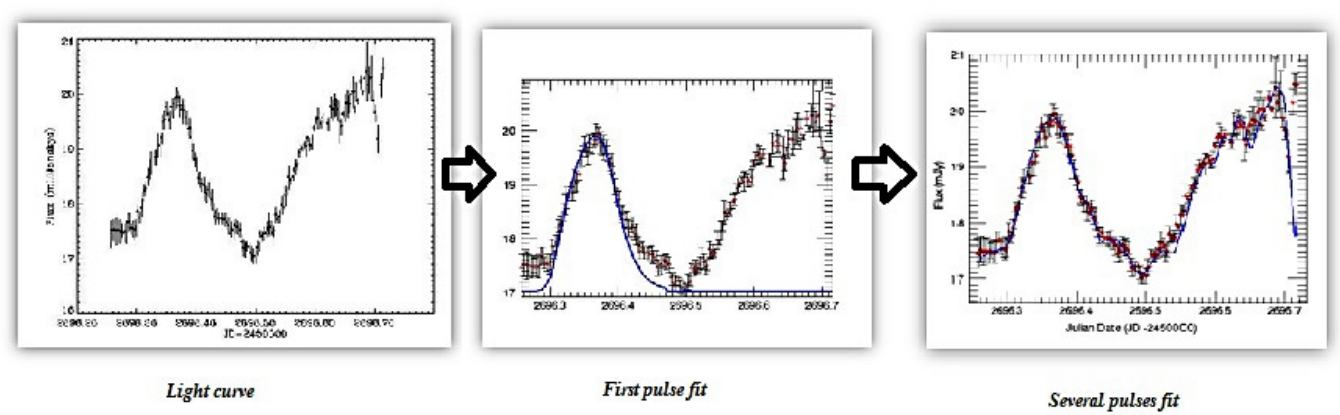

Figure 6. The process of fitting pulses to actual micro-variability curves. The light curve is an actual micro-variability curve of S5 0716+71 that has been linearized and fit with individual pulses. The process is currently done by eye, but will soon be automated using a smart fitting routine.

\section{Discussion}

The intermittency, the stochastic nature, and the similar profile shapes seen in micro-variations at different times and in different objects have led us to a possible model to explain the observed behavior. The model is based on a strong shock propagating down a relativistic jet and encountering turbulence, or conversely turbulent eddies propagating down the jet encountering a standing shock. By fitting pulses profiles generated from the model to our micro-variability observations, we obtain excellent fits to actual micro-variations. We fit the model to a 72-h micro-variability curve that was the result of a WEBT collaboration [13]. Thirty-five individual pulses were fit with a correlation coefficient 
of 0.97 . The fits resulted in cell sizes ranging from 9 AU to $165 \mathrm{AU}$ with an average of around $33 \mathrm{AU}$. The resulting cell size distribution was a continuum up to about $60 \mathrm{AU}$ consistent with what one would expect for a stochastic process. To show this model is applicable to other sources not on our list, the model was also fit to seven light curves of four quasars from the Kepler observatory archives. The objects Zw 229-15, 1925+50, 1858+48, and 1904+37 all showed significant micro-variability and the photometry was very high quality [14]. Each cadence averaged over 4200 data points to which an average of 42 pulses were fit. The correlation coefficients of the pulses were all over $99 \%$. The cell sizes ranged from 10 to $140 \mathrm{AU}$ for the same jet parameters and shock speeds.

A prediction of the model is that the pulses have different shapes depending on the frequencies observed, thus the spectral index should change as a function of pulse duration. To test the model, we have been looking for spectral index changes as a function of pulse duration predicted by the model using our ground-based SARA telescopes. Figure 7 shows a micro-variability curve of $0716+71$ taken on 3/4/2014 in the V and I filters. Four pulses were fit to the curve, and pulse three is shown in the bottom left panel mainly because it was the best sampled pulse of the four.

S5 0716_71 simultaneous V and I data from 3/4/2014

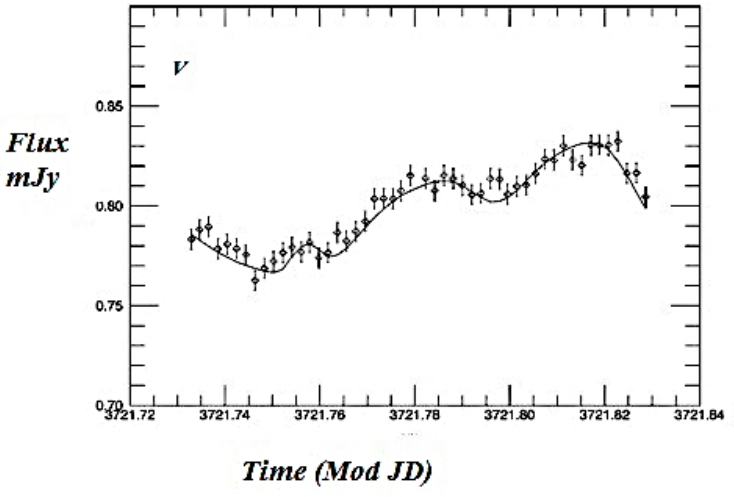

Relative Pulses \#3

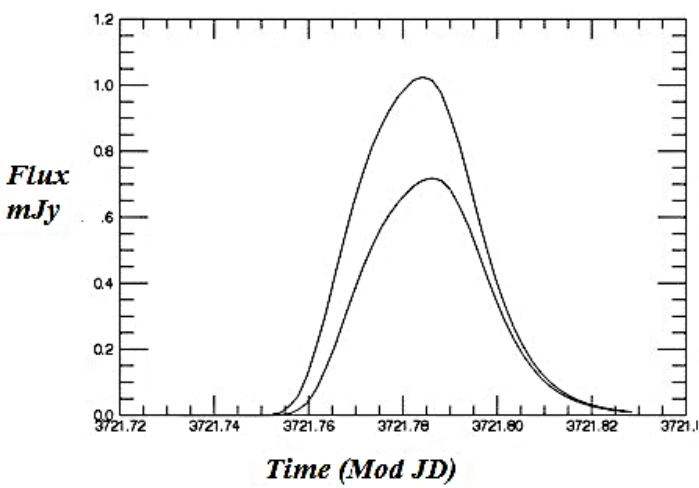

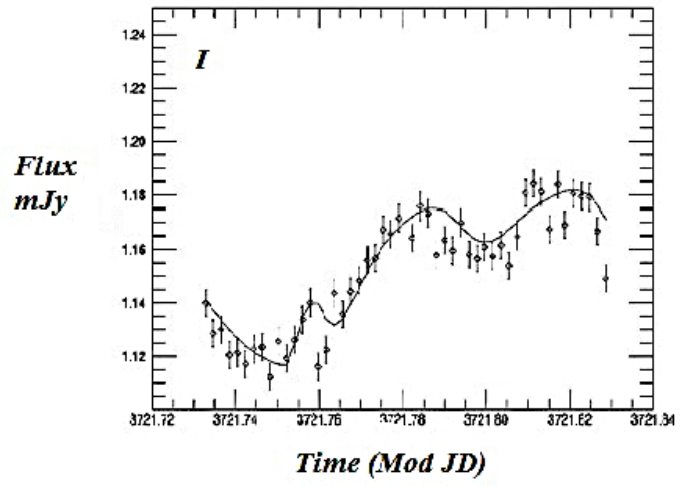

Spectral Index Evolution Pulse \#3

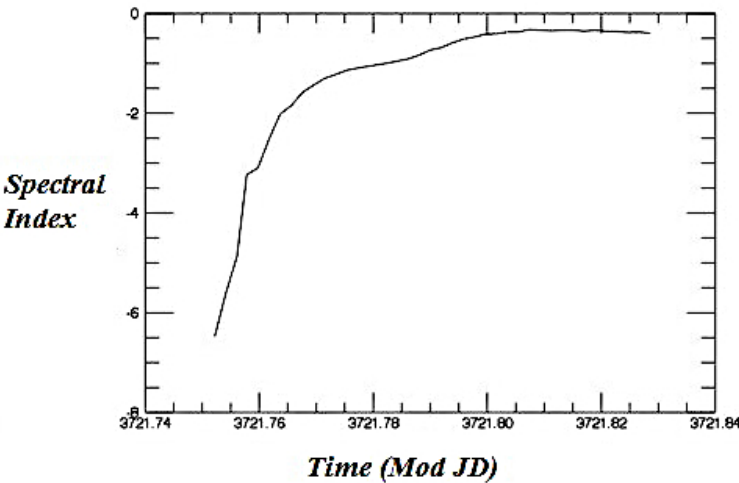

Figure 7. Simultaneous optical micro-variability light curves of S5 0716+71, taken at the SARA observatory at Kitt Peak on 3/4/2014. The upper left curve was through a V filter, while the right top curve was taken through an I filter. We independently fit each of the light curves to remove bias for center times/amplitudes. We are continuing to monitor these objects to try and get additional multi-frequency micro-variability curves to further test the model against observations.

Future observations and tests would be better accomplished with larger aperture telescopes; thus, we are looking into proposing to spend time on large aperture instruments to follow up our one-meter observations. A critical observation would be observing the polarization during these pulses. An ideal scenario is to get an observation on a large telescope equipped with a spectra polarimeter and observe 
one of our very active Blazars. An example would be the $10.4 \mathrm{~m} \mathrm{GCT}$ telescope equipped currently with CIRCE. With CIRCE we could measure the polarization and fluxes in the J, $\mathrm{H}$ and $\mathrm{K}$ bands simultaneously in a single observation. This would allow not only the pulse shape with frequency test but also the polarization changes.

\section{Conclusions}

The FIU/SARA Blazar micro-variability program has gathered over 12 years of observations in several optical frequency bands. Based on the analysis of this data, we have proposed a model for micro-variations which successfully explains the shape of the pulses de-convolved from the light curves, and also the characteristic intermittency of the occurrences of micro-variations. This model consists of an intermittent turbulent jet with a shock wave energizing individual turbulent cells within the jet. The micro-variations we observe are pulses of synchrotron radiation from these cooling turbulent cells. We are testing the model with further multi-frequency micro-variability observations and preliminary tests have been positive. We plan on using larger telescopes in the future to look for the subtle ( $\Delta \sim 0.005$ magnitude) spectral changes predicted by the model. We also discuss the use of polarization as a further test for the model. If the model is in fact correctly interpreting the micro-variations as detecting individual turbulent cells, then cell characteristics such as the size distributions and perhaps Reynolds numbers of the jet flow can be gleaned from the observations and used to inform other jet calculations.

Acknowledgments: This research is based on observations obtained with the SARA Observatory $0.9 \mathrm{~m}$ telescope at Kitt Peak, the 24-inch at Cerro Tololo in Chile, and the JKT at La Palma which are owned and operated by the Southeastern Association for Research in Astronomy (saraobservatory.org).

Conflicts of Interest: The authors declare no conflict of interest.

\section{References}

1. Habibi, F.; Moniez, M.; Ansari, R.; Rahvar, S. Simulation of optical interstellar scintillation. Astron. Astrophys. 2013, 552, A93. [CrossRef]

2. Pollock, J.T.; Webb, J.R.; Azarnia, G. Simultaneous Microvariability Observations of 0716+71. Astronom. J. 2007, 133, 487-488. [CrossRef]

3. Howard, E.S.; Webb, J.R.; Pollock, J.T.; Stencel, R.E. Microvariability and Long-Term Variability of Four Blazars. Astronom. J. 1994, 127, 17-23. [CrossRef]

4. Webb, J.; Bhatta, G.; Hollingsworth, H. Microvariability in Blazars. In Proceedings of the AAS 216th Meeting, Miami, FL, USA, 24-26 May 2010.

5. Dhalla, S.M.; Webb, J.R.; Bhatta, G.; Pollock, J.T. The Nature of Microvariability in Blazar $0716+71$. J. Southeastern Assoc. Res. Astron. 2010, 4, 7-11.

6. Azarnia, G.; Webb, J.; Velazquez, H. FIU-NSF Correlative Study Progress Report: Microvariability and Short-Term Variations in Blazars. In Proceedings of the ASP Conference, Florida International University, Miami, Florida, USA, 10-12 April 2005.

7. Bhatta, G.; Webb, J.R.; Hollingsworth, H.; Dhalla, S.; Khanuja, A.; Bachev, R.; Blinov, D.A.; Böttcher, M.; Calle, O.B.; Calcidese, P.; et al. The 72-h WEBT Microvariability Observation of blazar S5 0716+ 714 in 2009. Astron. Astrophys. 2013, 558, A92. [CrossRef]

8. Jones, T.W. Polarization as a probe of magnetic field and plasma properties of compact radio sources-Simulation of relativistic jets. Astrophys. J. 1988, 332, 678-695. [CrossRef]

9. Marscher, A.P.; Gear, W.K.; Travis, J.P. Variability of Nonthermal Continuum Emission in Blazars. In Variability of Blazars; Valtaoja, E., Valtonen, M., Eds.; Cambridge University Press: Cambridge, UK, 1992; p. 85.

10. McHardy, I. Explaining X-ray Variability in Blazars. In Proceedings of the Workshop on Blazar Variability across the EM Spectrum, Palaiseau, France, 22-25 April 2008.

11. Marscher, A.P.; Jorstad, S.G. Rapid Variability of Gamma-ray Emission from Sites near the $43 \mathrm{GHz}$ Cores of Blazar Jets. 2010, arXiv:1005.5551v1.

12. Kirk, J.G.; Rieger, F.M.; Mastichiadis, A. Particle acceleration and synchrotron emission in blazar jets. Astron. Astrophys. 1998, 333, 452-458. 
13. Bhatta, G.; Webb, J.R.; Hollingsworth, H.; Dhalla, S.; Khanuja, A.; Bachev, R.; Blinov, D.A.; Böttcher, M.; Calle, O.B.; Calcidese, P.; et al. The 72-h WEBT microvariability observation of blazar S5 0716+714 in 2009. Astron. Astrophys. 2013, 558, A92. [CrossRef]

14. Bhatta, G.; Webb, J.; Dhalla, S.; Hallingsworth, H. A Model for Microvariability in Blazars. In Proceedings of the AAS 218th Meeting, Boston, MA, USA, 22-26 May 2011.

(c) 2016 by the author; licensee MDPI, Basel, Switzerland. This article is an open access article distributed under the terms and conditions of the Creative Commons Attribution (CC-BY) license (http://creativecommons.org/licenses/by/4.0/). 